\title{
A 65-yr Climatology of Unusual Tracks of Tropical Cyclones in the Vicinity of China's Coastal Waters during 1949-2013
}

\author{
XUERONG ZHANG ${ }^{\text {a }}$ \\ Key Laboratory of Meteorological Disaster, Ministry of Education, and International Joint Laboratory on \\ Climate and Environment Change, and Collaborative Innovation Center on Forecast and Evaluation of \\ Meteorological Disasters, and Pacific Typhoon Research Center, Nanjing University of Information \\ Science and Technology, Nanjing, and State Key Laboratory of Severe Weather, Chinese Academy \\ of Meteorological Sciences, Beijing, China, and Department of Atmospheric and Oceanic \\ Science, University of Maryland, College Park, College Park, Maryland
}

\section{YING LI AND DA-LIN ZHANG}

State Key Laboratory of Severe Weather, Chinese Academy of Meteorological Sciences, Beijing, China, and Department of Atmospheric and Oceanic Science, University of Maryland, College Park, College Park, Maryland

\section{LIANSHOU CHEN}

State Key Laboratory of Severe Weather, Chinese Academy of Meteorological Sciences, Beijing, China

(Manuscript received 8 December 2016, in final form 16 October 2017)

\begin{abstract}
Despite steady improvements in tropical cyclone (TC) track forecasts, it still remains challenging to predict unusual TC tracks (UNTKs), such as the tracks of sharp turning or looping TCs, especially after they move close to coastal waters. In this study 1059 UNTK events associated with 564 TCs are identified from a total of 1320 TCs, occurring in the vicinity of China's coastal waters, during the 65-yr period of 1949-2013, using the best-track data archived at the China Meteorological Administration's Shanghai Typhoon Institute. These UNTK events are then categorized into seven types of tracks-sharp westward turning (169), sharp eastward turning (86), sharp northward turning (223), sharp southward turning (46), looping (153), rotating (199), and zigzagging (183) — on the basis of an improved UNTK classification scheme. Results show significant annual variability of unusual tracking TCs, ranging between 2 and 18 per year, many of which experience more than one UNTK event in the same or different UNTK types during their life spans. The monthly distribution of the UNTK events resembles that of TCs, with more occurring in June-November. An analysis of their spatial distributions reveals that all of the UNTK events tend to take place in the areas to the south of $30^{\circ} \mathrm{N}$, most frequently in the South China Sea and to the east of the Philippines. The results suggest that more attention be paid to the improved understanding and prediction of UNTK events so that the current positive trend in TC track forecast accuracy can continue for many years to come.
\end{abstract}

\section{Introduction}

According to the Eighth International Workshop on Tropical Cyclones (IWTC) of the World Weather Research Programme/Tropical Cyclone Programme (Elliott et al. 2014), the annually averaged position errors of

\footnotetext{
${ }^{a}$ Current affiliation: Jiangsu Institute of Meteorological Sciences, Nanjing, China.

Corresponding author: Prof. Da-Lin Zhang, dalin@umd.edu
}

operational tropical cyclone (TC) track forecasts have been reduced globally over the last decade. For the western North Pacific Ocean (WNP), 72-h forecast errors have improved from roughly $350 \mathrm{~km}$ in 2004 to about $200 \mathrm{~km}$ in 2014 , so today's 72-h track forecasts are better than 48-h forecasts in the early 2000s. Similarly, significant progress has been made in forecasting TC track and landfall location over the Atlantic Ocean basin during the past few decades (Rappaport et al. 2009). However, large year-to-year error variations still exist, which are attributable partly to varying annual occurrences of TCs and 
partly to challenges in forecasting unusual TC tracks (UNTKs), such as sharp recurving (Hodanish and Gray 1993; Wu et al. 2011b; Wu et al. 2012), sharp turning (Chan et al. 1980; Carr and Elsberry 1995; Wu et al. 2013), looping (Xu and Gray 1982), or meandering (Holland and Lander 1993) TC tracks. Moreover, Huang et al. (2015) found that three classical beta-advection models had little ability to predict sharp left- and right-turning TC tracks.

If a TC's track is a combination of any of the abovementioned UNTKs, it will likely be a disaster for the track forecast. Two such examples are Typhoons Wayne (1986) and Nat (1991) with the most UNTK types ever recorded (see Fig. 1). That is, during their 15-20-day life spans over the South China Sea (SCS) and coastal waters near Taiwan, they both made more than four obvious directional reversals as a result of changes in the environmental flows and interactions with other TCs (Chan and Lam 1989; Chan 1995; Peng et al. 1995). At the time the global and regional models could predict their tracks reasonably well when the larger-scale flows were well defined. However, large track forecast errors were generated when unusual directional changes occurred (e.g., through binary TC interactions), indicating a particularly challenging task in forecasting such UNTKs. Despite the several different forms of UNTKs, only a few limited studies of certain UNTKs have so far been conducted. They are briefly reviewed below.

\section{a. Sharp turning tracks}

Previous composite studies have focused more on the sharp turnings of TCs that are a fundamental type for UNTKs. Chan et al. (1980) indicated that a cyclonic (anticyclonic) environmental flow with large positive (negative) vertical wind shear could be a good indicator for a left (right) turning motion. Hodanish and Gray (1993) noted that a TC would begin a sharp recurving motion when mid- to upper-level zonal winds in the northwestern quadrant at $6^{\circ}$ latitude from the storm center shifted from an easterly to a westerly component, while a left-turning motion would occur when strong southwesterlies (ahead of a midlatitude trough axis) at $6^{\circ}$ to the north of a TC became weak and shifted back to an easterly component in the upper troposphere (i.e., between 250 and $300 \mathrm{hPa}$ ).

Interactions of TCs with monsoon systems provide unique conditions for sharp track changes. Carr and Elsberry (1995) were among the first to document sharp northward turnings of TCs that were embedded in monsoon gyres (MGs) over the WNP. In their study, the binary TC-MG interaction, a likely explanation for sharp track changes, was examined with a nondivergent barotropic model, in which a TC is allowed to coalesce

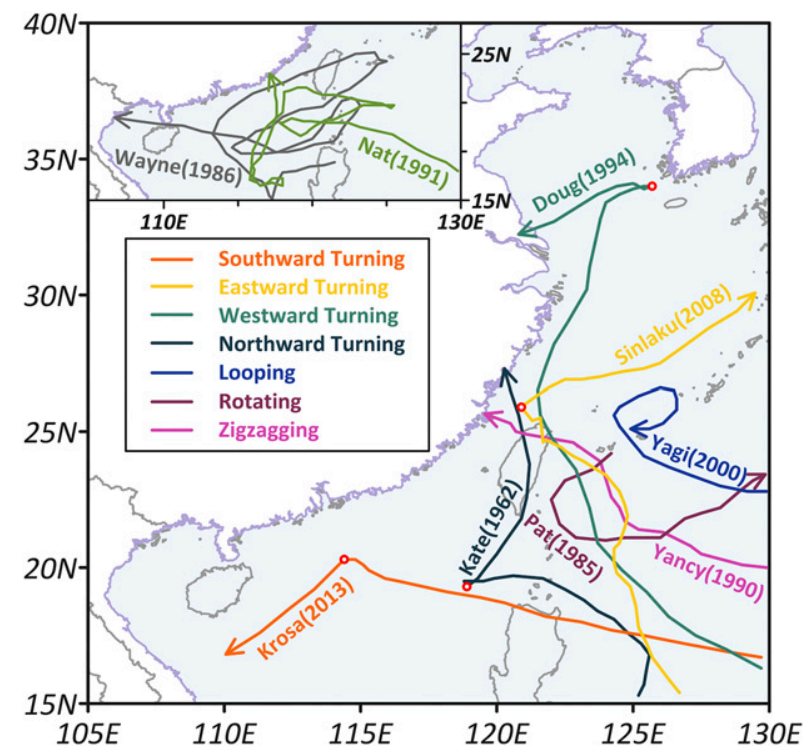

FIG. 1. Map of the study area, and samples of different UNTK types defined and analyzed in this study: TCs Krosa (2013), Sinlaku (2008), Doug (1994), and Kate (1962) are selected as a sample of sharp southward-, sharp eastward-, sharp westward-, and sharp northward-turning tracks, respectively (red open circles denote their sharp turning points); Yagi (2000), Pat (1985), and Yancy (1990) are selected as a sample of looping, rotating, and zigzagging tracks, respectively. Inset in the upper-left corner shows the UNTKs of TCs Wayne (1986) and Nat (1991) (see text).

with an MG. During the coalescence process, the $\beta$-induced dispersion of the MG produces strong ridging to the east and southeast of the coalesced system, resulting in a southerly flow that steers the TC northward sharply. Lander (1996) showed that approximately $80 \%$ of the TCs associated with reverseoriented monsoon troughs move on northward tracks. Monsoonal influences on TC westward and sharp northward turnings over the SCS and the WNP have been examined by filtering monsoon circulations into the time scales of the Madden-Julian oscillation (MJO), quasi-biweekly oscillation (QBW), and synoptic-scale flow (Wu et al. 2011a, 2013; Liang et al. 2011; Liang and $\mathrm{Wu} 2015$ ). Although the two types of turnings are closely associated with the interaction between low-frequency (i.e., MJO and QBW) and synoptic flows, the coalescence with the MG is crucial to the occurrence of sharp northward turnings, while TCs that take westward turnings do not experience such a coalescence process (Wu et al. 2011a; Liang et al. 2011; Liang and Wu 2015). Differing from the westward and sharp northward turnings, sharp northeastward turnings in the vicinity of the East China Sea could occur not only in an MJO-scale cyclonic gyre or a monsoon trough, but also at the bifurcation point of 
southeasterly and westerly steering flows (Wu et al. 2011b). Besides the monsoon circulation, subtropical highs (Wu et al. 2012; Shi et al. 2014), uppertropospheric cold lows (Wei et al. 2016), small lowlatitude anticyclones (Shi et al. 2014), and nearby TCs (i.e., binary interaction; Wu et al. 2003) could also play different roles in determining the sharp turnings of TCs.

\section{b. Zigzag tracks}

Unlike the sharp turning tracks, some TC tracks exhibit a "snake" or "S shaped" pattern, that is, turning left and shortly right or vice versa at least once while moving forward. This kind of UNTK, described as a tendency to meander or oscillate about a mean path, has also been called trochoidal oscillation (Yeh 1950; Kuo 1950, 1969; Jones 1977; Kitade 1981, Holland 1983; Willoughby 1988; Liu et al. 1999), zigzag (Chen and Ding 1979), or meandering motion (Holland and Lander 1993). Yeh (1950) and Kuo (1950) were the first to investigate the trochoidal oscillation of TC tracks. By comparing the vortex motion with the movement of a rotating solid cylinder in a uniform current on an $f$ plane, they both obtained a trochoidal path with a period depending on the intensity and size of the vortex, indicating that the trochoidal oscillation is similar to the Magnus effect, that is, a feature that can be seen when a spinning cylinder immersed in a fluid curves away from its original trajectory under a sidewise force (see Nof 1985). The subsequent theoretical work by Kuo (1969), after including frictional drags and lateral shear, showed that the period and lateral extent of the oscillation depend on both the rotation of the vortex and the mean flow speed, which was later confirmed by the numerical experiments of Jones (1977). However, through the use of simple divergent barotropic models on a $\beta$ plane, Kitade (1981) and Holland (1983) attributed the trochoidal oscillation to changes in vertical vorticity resulting from horizontal advection and the stretching process, rather than the Magnus effect. Based on the argument that deep convection could change the vertical vorticity of a TC through intense divergent flows, Willoughby (1988) obtained predominantly cyclonic track oscillations with periods between 2 and $10 \mathrm{~h}$ by imposing sinks and sources of mass into his barotropic model. Holland and Lander (1993) suggested that the presence of mesovortices or intense convective systems within a large TC could also lead to a meandering track with a period of several days. Lander (1996) discriminated TC track types, based on monsoonal flow patterns over the WNP, and found that $66 \%$ of S-shaped tracks are primarily associated with well-defined reverseoriented monsoon troughs.
It is well known that the orographic influence of an island, such as the island of Taiwan, can deflect a TC track (Yeh and Elsberry 1993a,b; Lin et al. 2006; M.-J. Yang et al. 2008; Huang et al. 2011; Peng et al. 2012; Wu et al. 2015). Yeh and Elsberry (1993a) showed that as the outer circulation of a TC begins to interact with a mountain barrier at approximately $250-400 \mathrm{~km}$ upstream, the mountain channeling effect may drift it southward. After the TC inner circulation hits the southern part of the barrier, its interaction with the barrier will deflect its track northward. Such successive terrain-induced track deflections could form an S-shaped path around the island, thus making it difficult to forecast its landfalling point.

\section{c. Looping tracks}

The looping track of a TC, defined as continuous directional changes on the same side of its previous course until forming a full circle, has attracted far less attention than have turning tracks. Xu and Gray (1982) was one of few studies focusing on the climatology and characteristic large-scale flow patterns associated with looping tracks. Based on a 21-yr (1957-77) survey of TC looping motions over the WNP, they found that looping TC tracks were always associated with a westerly trough to the northeast of TCs and that the more poleward the TC is relative to a subtropical high, the more westward the TC is relative to the westerly trough. Thus, the looping TC tracks are often embedded in weak environmental flows and far away from stronger flows ahead of westerly trough axes. As noted by Chen and Ding (1979) and C.-C. Yang et al. (2008), the mutual interactions of two or more TCs could also make the TC on the west side go through a looping motion.

It is evident from the review given above that previous studies have examined some types of UNTKs to a certain degree. However, few climatological studies have been performed to systematically document all types of UNTKs up to date in the vicinity of China's coastal waters (CCWs). Although Chen and Ding (1979) have conducted a 26-yr (i.e., 1949-74) climatology of some types of UNTK events occurring in the vicinity of CCWs, they focused more on the temporal characteristics of UNTKs with little attention to their spatial distributions. Similarly, while Xu and Gray (1982) examined a 21-yr (i.e., 1957-77) climatology of fast, slow, and looping TC motions over both the western Atlantic and the WNP, they focused mainly on the looping characteristics of UNTKs. Thus, the objectives of this study are to (i) provide an improved classification scheme that can be used to objectively distinguish UNTKs from the usual tracks of TCs occurring in the vicinity of CCWs and then classify them into various 
categories and (ii) perform an analysis of a 65 -yr (i.e., 1949-2013) climatology of the UNTKs with their quantitative spatiotemporal and morphological characteristics. Note that our study focuses more on the UNTKs occurring in the vicinity of CCWs, which covers the ocean areas from $15^{\circ}$ to $40^{\circ} \mathrm{N}$ and to the west of $130^{\circ} \mathrm{E}$ (see Fig. 1), instead of all of those over the entire WNP, because of the much-needed accurate forecasts for and rapid responses to unusual tracking TCs (UTTCs) that are fast approaching the coastlines of China and the other East Asian countries during the typhoon season. Through the present study, we wish to extend and update the previous studies of the UNTK climatology that were conducted nearly four decades ago by both Chen and Ding (1979) and Xu and Gray (1982). In particular, we wish to draw more attention to improving the understanding of multiscale processes (e.g., from monsoon circulations to subtropical highs, westerly disturbances, upper-tropospheric cold lows, orography, and deep convection) leading to the development of UNTKs that may threaten the environments and human beings along the coastal regions of China and the other East Asian countries.

The next section provides a review of the previous classification schemes for UNTKs. Section 3 describes the data source used for the present study and then presents an improved UNTK classification scheme. Section 4 shows the 65 -yr temporal characteristics of the seven types of UNTKs in the vicinity of CCWs, while section 5 presents the UNTKs' spatial distributions. A summary and concluding remarks are given in the final section.

\section{A review of previous classification schemes}

To systematically study the characteristics of UNTKs in the vicinity of CCWs, it is desirable to develop an objective classification scheme that can distinguish UNTKs from those usual ones. Previous studies have classified usual TC tracks into two categories: gradual recurving and straight moving, based on some empirical considerations, such as the west-to-northwestward movement or the north-to-northeastward recurving motion under the influences of steering flows associated with the WNP subtropical high (e.g., Visher 1922; Chu 1924; Riehl and Shafer 1944; Wu et al. 2012), cluster analysis (Camargo et al. 2007), and empirical orthogonal functional analysis (Liu and Chan 2008). In some studies, a gradual recurving track is also referred to as the hyperbola or parabola track (e.g., Visher 1922; Riehl 1954). By definition, gradual recurving TCs turn gradually northward or northeastward from their westward or northward tracks, whereas straight-moving
TCs move westward, northwestward, or northward in a straight track until they make landfall or dissipate over coastal waters or tropical oceans. Only Hodanish and Gray (1993) proposed a more specific criterion for gradual recurving TCs, that is, for those whose directional changes are at least $30^{\circ}$ (but less than $45^{\circ}$ ) to the right of their previous west-northwestward course within $36 \mathrm{~h}$ after passing the starting point of recurvature. Chan et al. (1980) defined the straightmoving track as a directional change of less than $10^{\circ}$ in $24 \mathrm{~h}$. The remaining tracks could be considered as UNTKs after classifying usual TC tracks with the abovementioned two definitions (Hodanish and Gray 1993; Chan et al. 1980).

Although some studies have attempted to classify UNTKs, a commonly accepted classification scheme for all UNTKs is still not available in the literature. Riehl and Shafer (1944) listed three types of unusual TC tracks: multiple recurving (i.e., a northward recurvature followed by a westward one), southeastward, and southwestward tracks. Later, Riehl (1954) added two more types to the abovementioned classification, that is, humping (or cusp) and looping tracks. After surveying TC tracks in the vicinity of CCWs from 1949 to 1974 , Chen and Ding (1979) classified UNTKs into seven types: westward turning over the Yellow Sea, northward turning over the SCS, inverted parabola, binary rotating, zigzag, clockwise looping, and counterclockwise looping. According to the abovementioned UNTK classifications, zigzag or multiple recurving tracks are similar to the trochoidal, meandering, or S-shaped tracks, while humping, cusp, or inverted parabola tracks are similar to the westward-turning tracks. Before developing a more comprehensive classification scheme for UNTKs, we first summarize the definitions of certain UNTKs from previous studies.

\section{a. Sharp turning tracks}

Chan et al. (1980) used a minimum directional change of $20^{\circ}$ in $12 \mathrm{~h}$ to define left- and right-turning tracks. Sharp recurving, which is also one type of the sharp turning tracks, was not precisely distinguished from gradual recurving until the study of Hodanish and Gray (1993), who defined left turning and sharp recurving as two types of unusual turning tracks, after comparing between gradual recurving and straight-moving tracks. They defined the sharp recurving tracks as those TCs changing their directions of motion for at least $45^{\circ}$ to the right of their previous west-northwestward course within $36 \mathrm{~h}$. They also quantitatively defined left-turning tracks, which have long been regarded as a type of UNTK, as a track displacing between $45^{\circ}$ and $130^{\circ}$ and then heading between $130^{\circ}$ and $200^{\circ}$ for at least $36 \mathrm{~h}$ each 


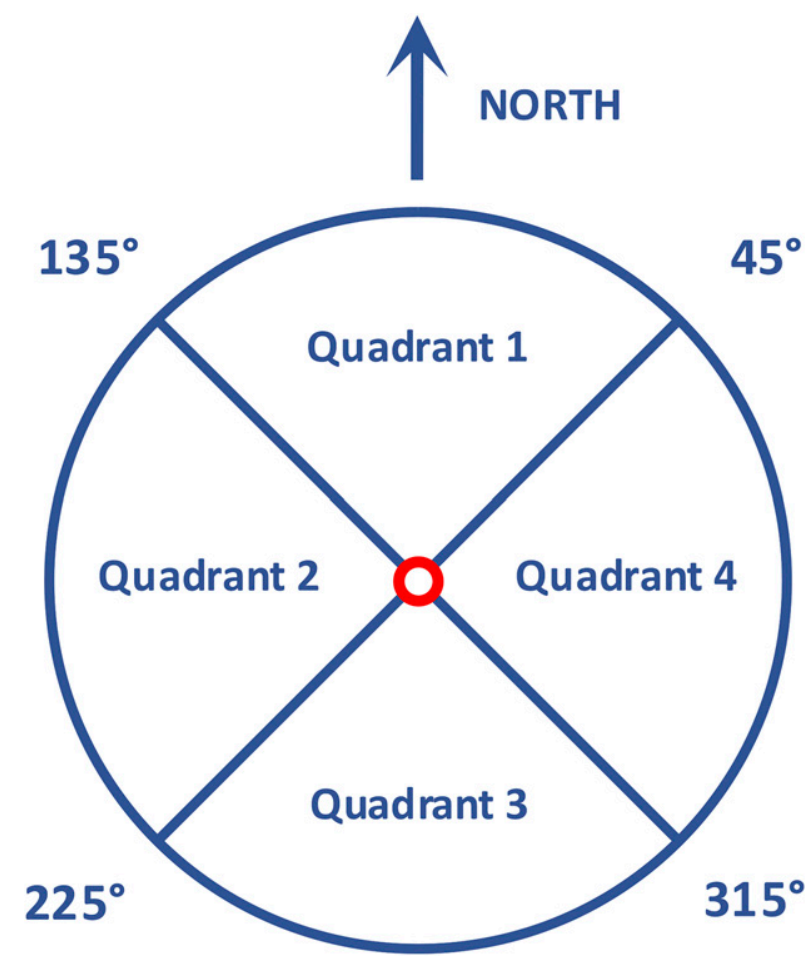

FIG. 2. Schematic diagram illustrating four quadrants (each spanning a $90^{\circ}$ tangential arc). Direction is measured counterclockwise from due east. Quadrant 1 points to true north. The red open circle denotes the turning point.

prior to reaching and after passing the left-turning point, respectively (Fig. 2). Wu et al. (2011b) defined sharp northeastward-turning tracks in the vicinity of the East China Sea as those turning $60^{\circ}$ over a $24-\mathrm{h}$ period and then replacing their northwestward movements by northeastward ones with a translation speed of less than $4.4 \mathrm{~m} \mathrm{~s}^{-1}$. In another study, Wu et al. (2013) defined a westward-turning track as a track with directional changes exceeding $25^{\circ}$ during $12-$ and $6-\mathrm{h}$ periods and a sharp northward-turning track as a track with directional changes exceeding $40^{\circ}$ in a 12 -h period and $37^{\circ}$ in a 6-h period.

\section{b. Zigzag tracks}

Providing a perfect definition for zigzag tracks is not straightforward, because they involve a wide range of scales and take on several different forms, including small-amplitude and short-period trochoidal oscillations around a relatively smooth track, large-scale and longerperiod meanders, and more erratic and nonperiodic meanders (Holland and Lander 1993). Yeh (1950) formulated the amplitude and period of trochoidal oscillations but a unified definition still has not been established, since the formulas, depending on the specific intensity and size of the storm, will vary for different storms and will need observational validation. Holland and Lander (1993) categorized track changes into three groups: synoptic track, medium-scale meanders (i.e., meanders with periods of greater than 1 day and an amplitude of several tens to hundreds of kilometers), and small-scale oscillations (i.e., oscillations with scales of around the TC eye diameter and periods of several hours). Using a combination of visual examination and second-order polynomial track smoothing, they found that the most frequent period was around $90 \mathrm{~h}$ with an amplitude of $50-100 \mathrm{~km}$ for the mediumscale meanders. In Lander (1996), an S-shaped track is defined as a northward motion of a TC that moves eastward at low latitudes, turns to the north or northwest, and eventually bends northeastward as the TC enters the midlatitude westerlies.

\section{c. Looping tracks}

$\mathrm{Xu}$ and Gray (1982) provided a detailed description of looping tracks by defining almost all the related characteristics: (i) the start and end times, at which a TC lost and regained a stable direction and speed; (ii) a period during which the TC is in a circular track; (iii) a diameter as the mean value of the long and short axes of the tracking ellipses; (iv) looping locations as the centers of the tracking circles; and (v) clockwise versus counterclockwise tracks.

From the review given above, it is obvious that the classifications and definitions of UNTKs in the previous studies are far from perfect and that they could not be used here for our objective statistical analysis. For example, Riehl's (1954) classification does not include unusual northward and eastward turnings, but it allows both southeastward and southwestward tracks to be separate categories. In Chen and Ding (1979), northward turnings are concerned only with TCs occurring over the SCS, while the westward-turning and inverted parabola tracks are treated as two separate types of UNTKs. Except for the westward-, eastward-, and northward-turning tracks, which have both qualitative and quantitative definitions in the previous studies, zigzag and looping tracks have only qualitative definitions, and southward-turning and rotating tracks do not even have specific definitions. In short, to our knowledge, few studies have developed a comprehensive classification scheme for all types of UNTKs, although certain types of UNTKs have attracted significant attention. Thus, to facilitate our statistical analysis of the UNTKs in the vicinity of CCWs, we developed a UNTK classification scheme, based on various definitions used by the abovementioned studies. It is described in the next section. 


\section{Data source and methodology}

\section{a. Data source}

In this study, we use the best-track data at 6-h intervals for TCs occurring in the vicinity of CCWs during the years of 1949-2013 that were archived at the China Meteorological Administration's Shanghai Typhoon Institute (CMA STI) (http://tcdata.typhoon.org. cn/en/index.html); see Ying et al. (2014) for more details. The dataset includes TC position (latitude and longitude), minimum sea level pressure near the TC center, and intensity. Other variables (e.g., turning angle and loop diameter) can be deduced from the best-track data, for example, following Chan et al. (1980) and Xu and Gray (1982). This dataset has been previously used to study various aspects of TC tracks, including unusual TC motions (e.g., Zhao et al. 2012; Li et al. 2013; Wei et al. 2016; Shi et al. 2014). Figure 1 shows our study area. Note that UNTKs over the mainland will be excluded, because of some different processes leading to track changes from those over oceans. However, the Philippines, Hainan, Taiwan, and other islands are the constituent parts of the study area. We have noted some differences in TC positions from the best-track datasets archived at the CMA STI and Joint Typhoon Warning Center (JTWC), some of which have also been found by Barcikowska et al. (2012) and Ying et al. (2014). We do not think that the use of the JTWC's best-track data would affect significantly the identification of UNTK events as long as individual TC positions were systematically determined. In addition, the spatial scales of UNTKs are statistically larger than the TC position differences from the two datasets.

Note that the CMA STI best-track dataset contains tracks of all TC intensities, including tropical depression (TD). TDs (both named and unnamed) are used to build a comprehensive climatology of UNTKs in this study because (i) UNTKs are common in weak TCs or TDs (Xu and Gray 1982; Hodanish and Gray 1993; Burroughs and Brand 1973; Carr and Elsberry 1995; Wei et al. 2016); (ii) including TDs would help identify some UNTKs, as they require continuous directional changes at several track points; and (iii) once a TD develops in the SCS, it could quickly hit south China, Vietnam, and the Philippines, which would be of great concern to local TC forecasters and hazard mitigation managers. Although TDs tend to have larger position uncertainties than the other TC intensities-for example, $75 \mathrm{~km}$ for TDs occurring over the Atlantic and eastern Pacific basins (Torn and Snyder 2012) — they are much smaller than the average spatial scale of any UNTK type defined in this study, as will be discussed later.
One may question our use of best-track data prior to the satellite era for the present study, because the quality of best-track records has markedly improved after geostationary satellite observations became available since the early 1980s (Kossin et al. 2013; Ying et al. 2014). To see whether the changes in the best-track data quality would have any significant impact on our statistical analysis of UNTK events, we have compared their regional distributions between the periods of 1949-80 and 1981-2013. Results show that (i) a total of 556 and 503 UNTK events occurred during the abovementioned two periods, respectively; (ii) the annual distribution of UNTK events prior to and after 1980, to be shown in section 4 , does not show significant differences, just like their total events; and (iii) only some small-scale differences in spatial distribution between them could be noted (not shown). Thus, it was concluded that including the best-track data during 1949-80 affects little the statistical analysis results to be presented in sections 4 and 5 .

\section{b. An improved UNTK classification scheme}

After analyzing UNTKs occurring in the vicinity of CCWs during the 65-yr period of 1949-2013 using the abovementioned dataset, we may categorize them into the following seven types: sharp westward turning, sharp eastward turning, sharp northward turning, sharp southward turning, looping, rotating, and zigzag, based mostly on their track patterns. Figure 1 shows a sample of each UNTK type. Generally, a sharp turning track refers to a TC changing its direction of motion sharply only once within a certain period, whereas the last three types require continuous directional changes. More detailed descriptions of our classification scheme are given below.

\section{1) SHARP WESTWARD-, EASTWARD-, NORTHWARD-, AND SOUTHWARD-TURNING TRACKS}

The location where a TC changes its direction of motion sharply is the point of primary importance in the context of sharp turning tracks, which is referred to here as the sharp turning point. So, a sharp turning is determined by two factors: a pronounced turning angle and a directional change between different quadrants (Fig. 2). Specifically, the turning angle, defined here as an angle between two 6-hourly motion vectors before and after a turning point, should be at least $45^{\circ}$ for a sharp eastward turning, and more than $30^{\circ}$ for a sharp westward, northward, and southward turning. The turning angles of $30^{\circ}$ and $45^{\circ}$ are used herein because they correspond to the respective 85th and 93th percentiles of turning angles for all the TC cases used in this study. Moreover, as mentioned in section 2, gradual 
recurvings - that is, with directional changes of less than $45^{\circ}$ in $36 \mathrm{~h}$-are common over the WNP (Hodanish and Gray 1993), and this climatological feature can result in an increased count of eastward turning. Thus, the turning-angle criterion of sharp eastward-turning tracks (i.e., $45^{\circ}$ in $12 \mathrm{~h}$ ) is set higher than that of sharp westward-, northward- and southward-turning tracks (i.e., $30^{\circ}$ in $12 \mathrm{~h}$ ) to ensure that those selected eastward turnings are sharp enough for being considered as UNTKs and different from gradual recurving ones.

The directional change between different quadrants requires that a sharp westward turning will move on a course between $225^{\circ}$ and $135^{\circ}$ (i.e., in quadrants 3,4 , and 1) for more than $24 \mathrm{~h}$ prior to reaching its turning point, and then take a heading between $135^{\circ}$ and $225^{\circ}$ (i.e., in quadrant 2) for at least $12 \mathrm{~h}$ after passing the turning point. Similarly, a sharp eastward, northward, and southward turning will track on a heading between $45^{\circ}$ and $315^{\circ}$, between $135^{\circ}$ and $45^{\circ}$, and between $315^{\circ}$ and $225^{\circ}$, respectively, for at least $24 \mathrm{~h}$ prior to reaching their individual turning points, and then resume a course in quadrants 4,1 , and 3 , respectively, for more than $12 \mathrm{~h}$ after passing the turning points. Over the $36-\mathrm{h}$ period, other directional changes should be smaller than the angle change at the turning time, and the TC's movements should be relatively stable before and after the turning time.

\section{2) LOOPING AND ROTATING TRACKS}

Looping is defined to follow closely the definition of $\mathrm{Xu}$ and Gray (1982), including the start and end times, period, location, and direction, except that loop diameter is defined as the distance between the two most remote track points within $6^{\circ}$ latitude or less. This diameter of $6^{\circ}$ latitude is selected herein because it is close to the typical size of a TC. Open circle tracks of any diameter are all grouped into rotating tracks.

\section{3) ZigZAG TRACKS}

A segment of TC track is called the zigzag track if (i) a TC turns left and right or vice versa sequentially at least once while moving forward, (ii) the next directional change occurs within $30 \mathrm{~h}$ after the previous one, and (iii) the directional change at the inflection point is at least $30^{\circ}$ in azimuth over a 12 -h period. Based on the abovementioned three criteria, a selected zigzag track will change its motion direction frequently within a relatively short period and exhibit obvious oscillations about its mean track. Meanwhile, considering TC position uncertainties in the best-track data (Torn and Snyder 2012), single-peak oscillations with scales of less than $1^{\circ}$-latitude distance are excluded.

After applying the abovementioned classification scheme to the CMA STI's 65-yr best-track data, we find
564 of 1320 TCs (i.e., 43\%) in the study area that exhibited one or more UNTK events, which gives a mean of 8.7 UTTCs per year. The total (annual average) numbers of sharp westward-turning, sharp eastwardturning, sharp northward-turning, sharp southwardturning, and looping, rotating, and zigzagging UTTCs, as listed in Table 1, are 160 (2.5), 84 (1.3), 219 (3.4), 45 (0.7), 137 (2.1), 172 (2.6), and 177 (2.7) during 1949-2013, which account for $12 \%, 6 \%, 17 \%, 3 \%, 10 \%$, $13 \%$, and $13 \%$ of the 65 -yr total TCs, respectively. Their temporal variability and spatial characteristics are analyzed in sections 4 and 5, respectively.

\section{Temporal characteristics of UNTKs}

\section{a. Annual variability}

Figure 3a shows the annual number distributions of UTTCs (orange bars), which range from 2 (of 14) in 1983 to 18 (of 24) in 1994, and total TCs (blue bars), while Fig. 3b shows the annual percentage of UTTCs with respect to total TCs (dashed lines), which ranges from $14 \%$ ( 2 of 14 ) in 1983 to $75 \%$ in both 1991 (15 of 20) and 1994 (18 of 24). Significant annual variability can be seen in the numbers of both total TCs and UTTCs. Note that since a TC may experience more than one type of UNTK during its lifetime in the study area, the total numbers, percentages, and annual means of TCs for the sum of the seven UNTK types can exceed $564,43 \%$, and 8.7 UTTCs, respectively.

Figure $3 \mathrm{~b}$ also shows the annual event distribution of UNTKs (solid line). A total of 1059 UNTKs are found from 1320 TCs, with a mean event of 16.3 per year, 0.8 per TC, and 1.9 per UTTC. The annual event of UNTKs ranges between 2 in 1983 and 35 in 1986. The total (annual average) events of sharp westwardturning, sharp eastward-turning, sharp northward-turning, sharp southward-turning, and looping, rotating, and zigzagging UNTKs, as listed in Table 1, are 169 (2.6), 86 (1.3), 223 (3.4), 46 (0.7), 153 (2.4), 199 (3.1), and 183 (2.8), respectively. Moreover, since a TC may experience the same type of UNTK more than once, the total (and the annual average) events of UNTKs are a little larger than the total (and the annual average) numbers of UTTCs for each UNTK type.

Figures $4 \mathrm{a}$ and $4 \mathrm{~b}$ show the distributions of TCs as a function of UNTK types and events, respectively. Evidently, most UTTCs are involved with only one type of UNTK (55\%, i.e., 310 of 564) or even one UNTK event (54\%, i.e., 306 of 564$)$. However, our analyses do reveal the presence of TCs with more than one type of UNTK or with more than one UNTK event. The results indicate that the former compose $19 \%$ of total TCs and $45 \%$ of UTTCs (i.e., 254 of 1320, and 564, respectively, where 


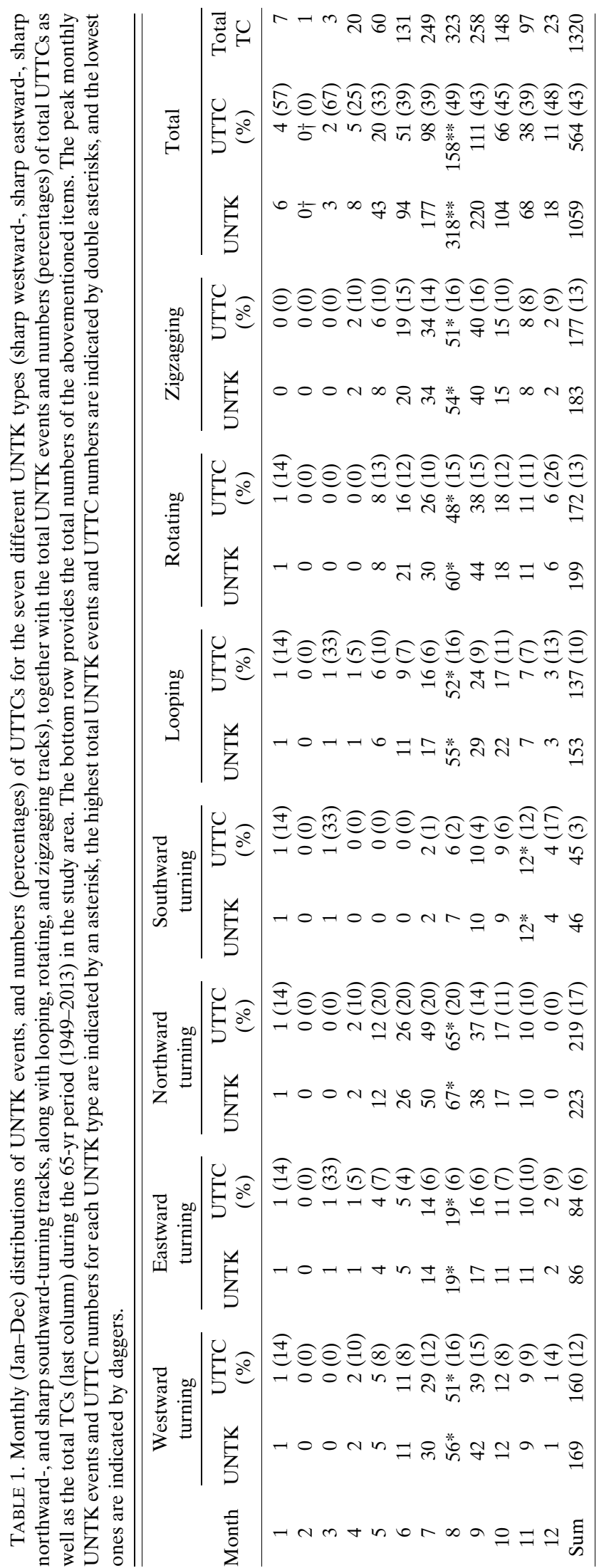



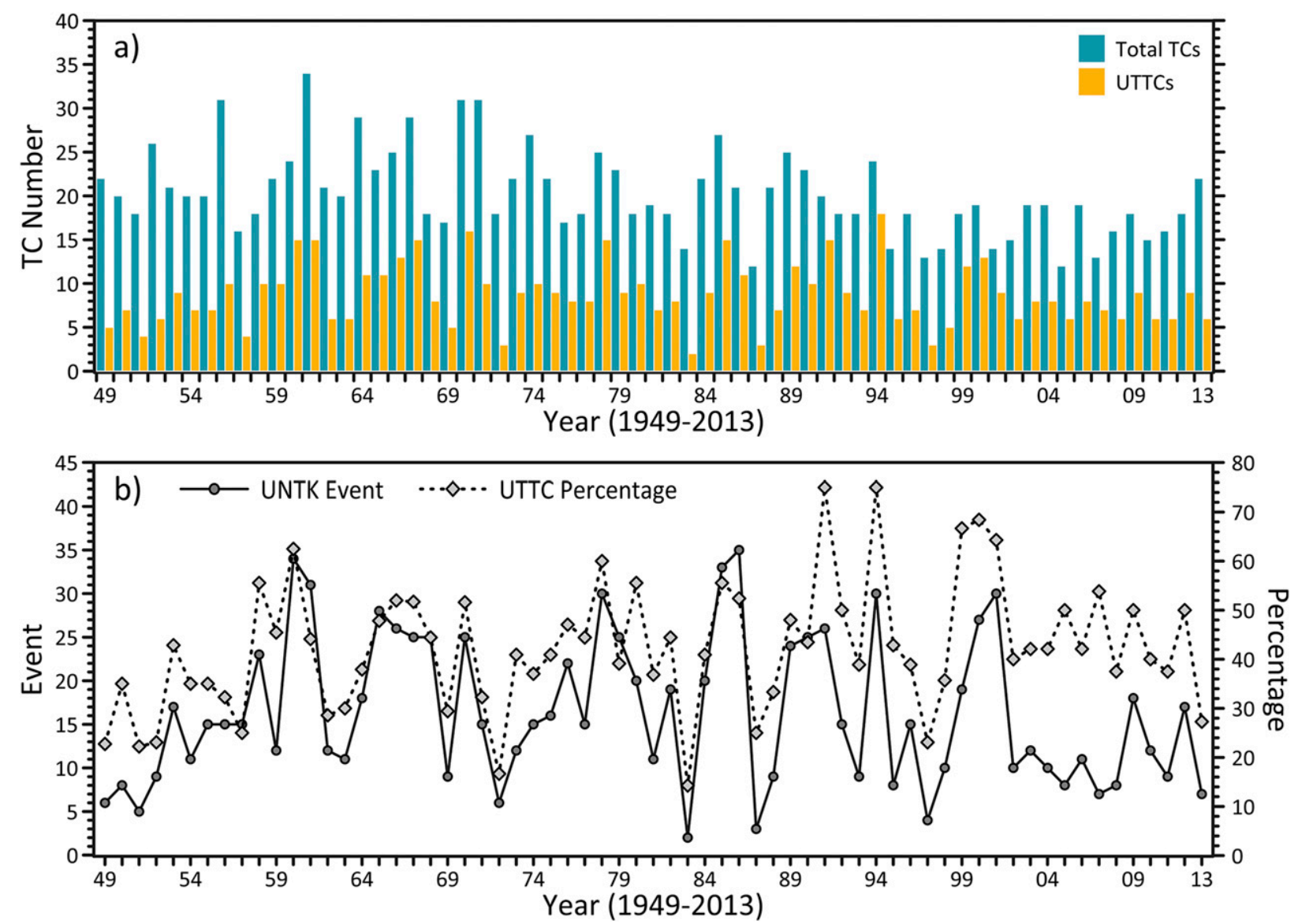

FIG. 3. Annual distributions of (a) the number of UTTCs (orange bars) and total TCs (blue bars), and (b) the UTTCs as a percentage of total TCs (dashed lines with open diamonds) and the events of UNTKs (solid line with circles) in the study area during the 65-yr period of 1949-2013.

254 is the sum of $142,69,26,13$, and 4 in Fig. 4a), while the latter compose $20 \%$ of total TCs and $46 \%$ of UTTCs (i.e., 258 of 1320 and 564, respectively, where 258 is the sum of 137, 65, 28, 14, 6, 3, 1, 3, and 1 in Fig. 4b). It is obvious that some TCs may experience more than one UNTK event of the same or different UNTK types during their life spans, which also gives a reasonable explanation for the relative high percentage of UTTCs in 1320 TCs (see dashed lines in Fig. 3b). In this regard, it is of interest to note that Typhoon Wayne (1986) experienced 10 UNTK events involving six different UNTK types, namely, two sharp westward turnings, two sharp southward turnings, one sharp eastward turning, one sharp northward turning, three rotating tracks, and one zigzag track (see Fig. 1).

\section{b. Monthly variability}

Figure 5a shows the monthly distributions of total UTTCs (orange bars) as compared with total TCs (blue bars), and the monthly total UTTCs as a percentage of total TCs (denoted by the number above each orange bar), while Figs. 5b and 5c present the monthly distributions for the seven types of UNTKs; all of which are also given in Table 1 . Note that a TC may exhibit several UNTK events entailing portions of two consecutive months, which is common from our climatological analysis. Typhoon Wayne (1986) was also a good example in this respect, in which the first 9 UNTK events occurred in August with its 10th in September. Thus, for the sake of consistency, all the UTTC numbers and UNTK events associated with the same TC, as shown in Figs. 5a-c and Table 1, are counted only in accordance with the month it appeared first in the study area.

It is evident that like total TCs, (i) total UTTCs appeared mainly in June-November with many more events occurring in July-September, and (ii) they barely appeared in January-April. The higher probability of UTTCs in July-September may be attributed partly to their more corresponding TC occurrences and partly to complex influences of multiscale circulations, for example, as noted by Carr and Elsberry (1995), Wei et al. (2016), and Lander (1996). The UNTK events and 

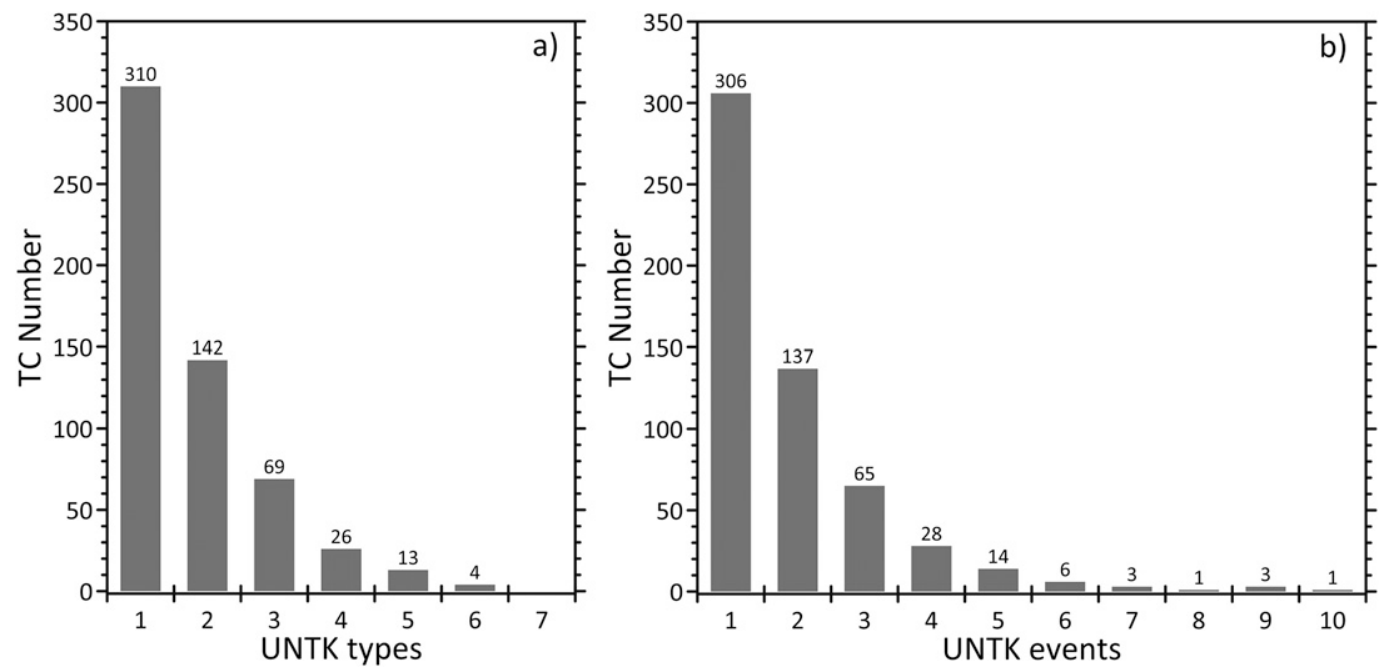

FIG. 4. Number distributions of TCs, given above each bar, as a function of (a) UNTK types and (b) UNTK events in the study area during the 65-yr period of 1949-2013.

UTTC numbers of the seven UNTK types all had a similar monthly distribution. Evidently, both the total events of UNTKs (adding all the bars by the months in Figs. 5b,c) and the numbers of total UTTCs peaked in August at 318 and 158 (as indicated by double asterisks in Table 1), respectively, and February is the only month without any UNTK and UTTC (as indicated by a cross in Table 1). In addition, for a single UNTK type, except for the sharp southward-turning track reaching the peak UTTC numbers and UNTK events in November, all the other six types peaked in August (see Figs. 5b,c, and as indicated by an asterisk in Table 1).

\section{Spatial characteristics of UNTKs}

Figure 6 shows the spatial distribution of seven different types of total UNTKs in three different ways [i.e., (i) track segments; (ii) sharp turning points (Figs. 6a-d), or geometric centers of looping and rotating tracks (Figs. 6e,f), or inflection points of zigzagging tracks (Fig. 6g); and (iii) the densities of the points or geometric centers within $2.5^{\circ} \times 2.5^{\circ}$ grids] over the study area during 1949-2013. They are discussed below in three groups: one for sharp turning UNTKs, one for looping and rotating ones, and the third one for zigzag tracks. Despite their differences in morphology and movements, a common feature is that all the seven types of UNTKs tended to take place in the areas to the south of $30^{\circ} \mathrm{N}$, most frequently in the SCS. The latter indicates that more attention should be paid to forecast improvements of UNTKs over the SCS, given the current challenges in forecasting UNTKs.

\section{a. Sharp westward-, eastward-, northward-, and southward-turning tracks}

It is evident from Figs. 6a-d that 24-h sharp westwardand northward-turning tracks (i.e., $12 \mathrm{~h}$ before and $12 \mathrm{~h}$ after the sharp turning points) tended to cluster to the east of the Philippines (9-12 events over some grids), in addition to their major clustering (14-15 events over some grids) over the SCS, and that many fewer sharp eastward- and southward-turning events appeared in the two areas relative to the other two types of sharp turning events. In fact, few sharp southward-turning events took place to the north of $30^{\circ}$ (Fig. 6d). Given their frequent UNTK events in the SCS, sharp westward- and northward-turning TCs in this region could quickly hit Vietnam or southern China (Figs. 6a,c) and catch them off guard, likely resulting in disasters. On the other hand, some sharp eastward- and southward-turning TCs over the SCS (Figs. 6b,d), albeit with many fewer UNTK events, may cause the false alarms of TCs that are on the verge of landing in Vietnam or southern China and instead lead to disasters in Taiwan and the Philippines. The relatively active TC-MG interaction is a likely explanation for the higher sharp turning events over the SCS (Carr and Elsberry 1995; Wu et al. 2011a, 2013; Liang et al. 2011; Liang and Wu 2015).

Although fewer sharp turning TCs are expected to occur while they move into higher latitudes because of more dominant westerly flows, sharp turning TCs over the Yellow Sea and the East China Sea could be of great concern from a forecasting standpoint. Since they are surrounded by China, the Korean Peninsula, and Japan, any $\mathrm{TC}$ with a sharp turning in any direction may be 

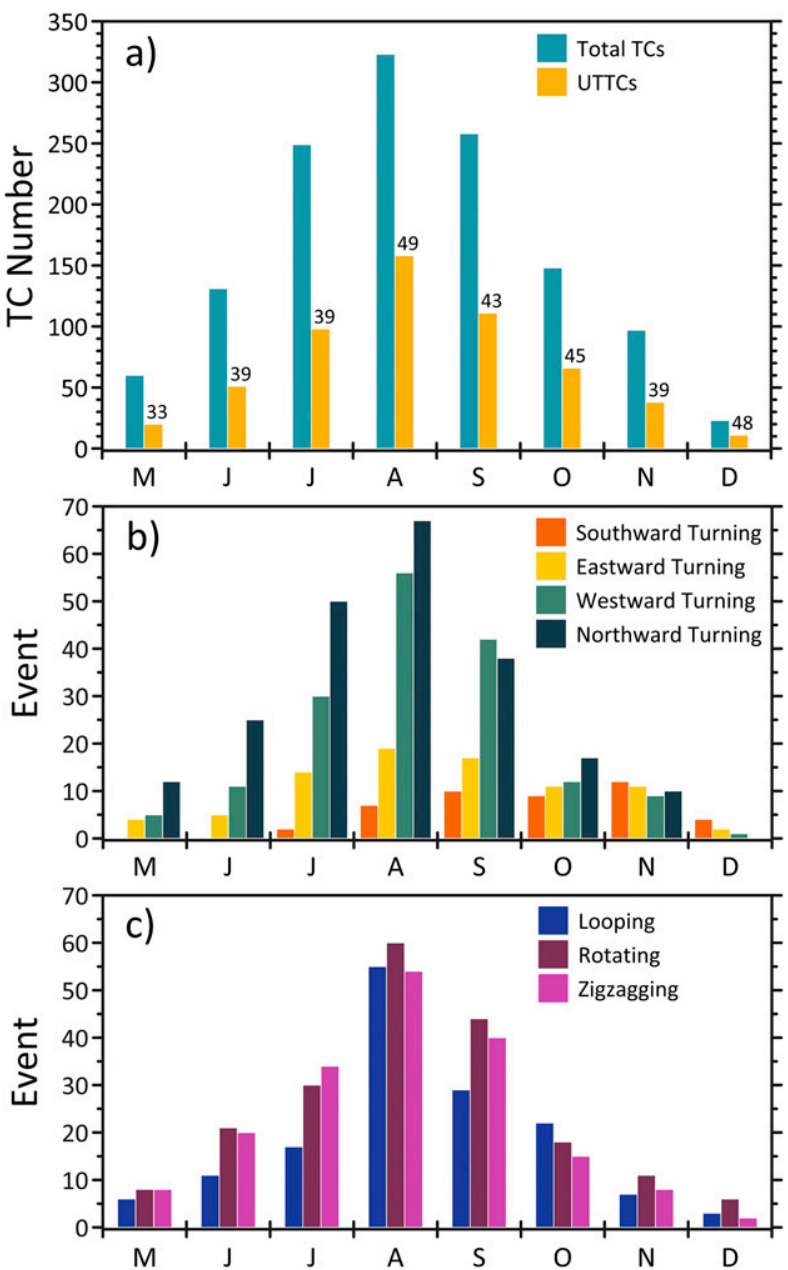

FIG. 5. Monthly distributions of (a) the total TCs (blue bars) and the total UTTCs (orange bars); (b) the events of sharp southward-, sharp eastward-, sharp westward-, and sharp northward-turning tracks; and (c) the events of looping, rotating, and zigzagging tracks in the study area during the 65-yr period of 1949-2013. The number above each orange bar in (a) indicates the percentage of UTTCs for each month.

more likely to hit one of the countries eventually and make a false alarm in the other countries. Thus, sharp turnings, which may cause devastating damage to coastal countries, are of great forecasting importance.

To further reveal their turning characteristics, especially those occurring over coastal waters, sharp turnings are stratified by turning angles between $30^{\circ}$ and $60^{\circ}$, between $60^{\circ}$ and $90^{\circ}$, and between $90^{\circ}$ and $180^{\circ}$, as denoted by the blue, green, and red lines (and dots) with arrows, respectively, in Figs. 6a-d. Most sharp turnings had turning angles of $30^{\circ}-60^{\circ}$ (i.e., $66 \%$ or 343 of 523 ; see Table 1), but a notable portion turned more than $90^{\circ}$ (i.e., $16 \%$ or 86 of 523 ). Of interest is that most of the latter events also took place over the SCS, and that the sharper the turnings, the more slowly these TCs tended to move (i.e., their 24-h tracks are shorter).

\section{b. Looping and rotating tracks}

Figure 6e shows looping tracks that are shaped as closed circles of no larger than $6^{\circ}$ latitude in diameter. Note that few looping tracks are perfectly round; most are elongated or polygon shaped. It is evident from Fig. 6e that, like sharp westward and northward turnings, looping tracks also tend to cluster to the east of the Philippines and Taiwan, besides their major clustering (7-10 events over some grids) over the SCS. Although there were many fewer clockwise events in the areas to the north of $20^{\circ} \mathrm{N}$, clockwise (red lines and dots) and counterclockwise (blue lines and dots) looping tracks were similar in the spatial distribution. This is consistent with a survey of looping tracks over the WNP from 1957 to 1977 by $\mathrm{Xu}$ and Gray (1982), who also found the highest percentage of looping tracks to the south of $20^{\circ} \mathrm{N}$, with three centers taking place to the east of Taiwan and the Philippines, and in the northern part of the SCS, respectively. Xu and Gray (1982) attributed the reduced looping phenomenon to the increased westerly steering flows as they moved toward higher latitudes.

Given the significant percentage of looping and rotating TCs in the total TCs (i.e., 20\%) and UTTCs (i.e., $46 \%$ ) (see Table 1), it is worthwhile to describe briefly their morphologies in terms of direction and diameter as well as time scale. A close analysis of Fig. 6e reveals that out of 153 looping tracks, 107 (70\%) were counterclockwise and $46(30 \%)$ were clockwise. This is consistent with the finding of Xu and Gray (1982), who showed that counterclockwise looping tracks were more frequent than clockwise ones by a ratio of 2 to 1 in the WNP. The looping diameters ranged from $0.1^{\circ}$ (e.g., a small loop over Okinawa Island associated with Nari of 2001; Wu et al. 2008; Zhang et al. 2011) to 5.63 (e.g., Agnes of 1971 , centered at $20.7^{\circ} \mathrm{N}, 129.6^{\circ} \mathrm{E}$ ). Far more than half of the looping tracks $(80 \%$, i.e., 123 of $153)$ were less than $2^{\circ}$ in diameter. Thus, the average looping diameter is $1.34^{\circ}$, which is close to $1.4^{\circ}$ found by $\mathrm{Xu}$ and Gray (1982). The average diameter for the clockwise looping track was $1.6^{\circ}$, which was larger than that of the counterclockwise one (i.e., $1.2^{\circ}$ ). Because of the different diameters, the looping periods ranged from the shortest $18 \mathrm{~h}$ (e.g., Lekima from 2200 UTC 26 to 1600 UTC 27 September 2001) to the longest $174 \mathrm{~h}$ (e.g., Jean from 0600 UTC 8 to 1200 UTC 15 November 1962), with an average looping period of $37.3 \mathrm{~h}$, which is shorter than the average period of $48 \mathrm{~h}$ found by $\mathrm{Xu}$ and Gray (1982). About $90 \%$ of looping tracks can be completed in $72 \mathrm{~h}$. 

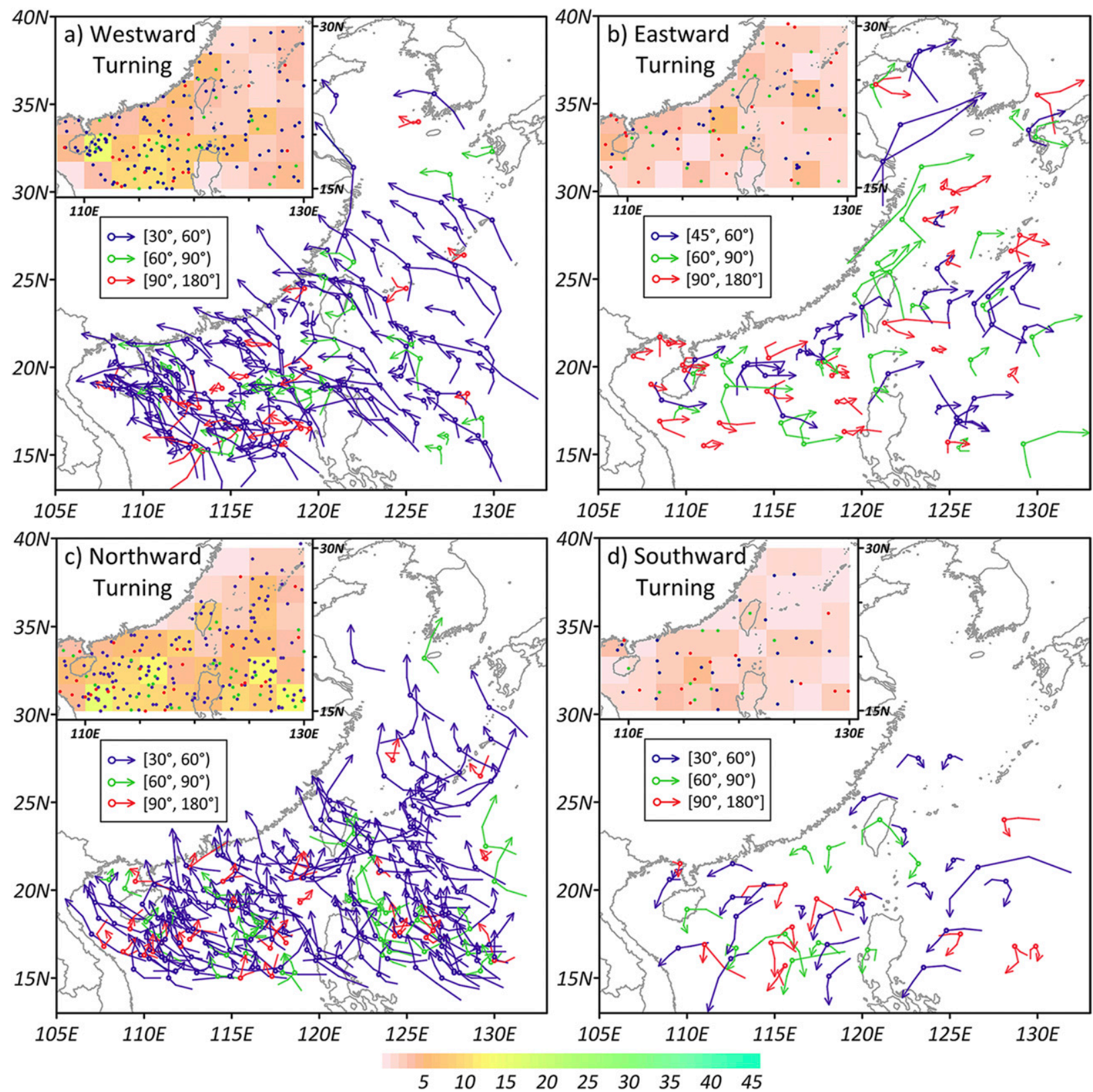

FIG. 6. Spatial distributions of (a) sharp westward-turning, (b) sharp eastward-turning, (c) sharp northward-turning, (d) sharp southward-turning, (e) looping, (f) rotating, and (g) zigzagging tracks during the 65-yr period of 1949-2013. In (a)-(d), blue, green, and red open circles and lines denote sharp turning points and 24-h tracks (i.e., $12 \mathrm{~h}$ before and $12 \mathrm{~h}$ after the sharp turning points) of sharp turnings with turning angles of less than $60^{\circ}, 60^{\circ}-90^{\circ}$, and greater than $90^{\circ}$, respectively; track arrows denote TC motion directions $12 \mathrm{~h}$ after sharp turnings. Open (filled) circles in (e) represent the start points of clockwise (counterclockwise) looping tracks, with red (blue) lines and dots denoting their respective tracks and geometric centers. The same information for rotating tracks is shown in (f). In (g), blue and red lines (dots) represent westward- and northward-moving zigzag tracks (inflection points), respectively. In top-left inset maps, dots denote sharp turning points in (a)-(d), geometric centers in (e) and (f), and inflection points in (g) with the same color conventions as those associated with their corresponding tracks. Color shadings indicate the densities of the dotted points within $2.5^{\circ} \times 2.5^{\circ}$ grids, with color scales given underneath (a)-(d) and (g).

Rotating tracks, which are shaped as open circles of any diameter, are given in Fig. 6f, showing much denser concentrations (i.e., $12-15$ events over some grids) than looping tracks over the SCS and within the longitudes of $125^{\circ}-130^{\circ} \mathrm{E}$. Like the looping tracks, rotating tracks also consist of clockwise (red lines and dots) and counterclockwise (blue lines and dots) ones, with a ratio of about 1 to 1.3 . Their average diameter was $3^{\circ}$, with $\pm 0.1^{\circ}$ 

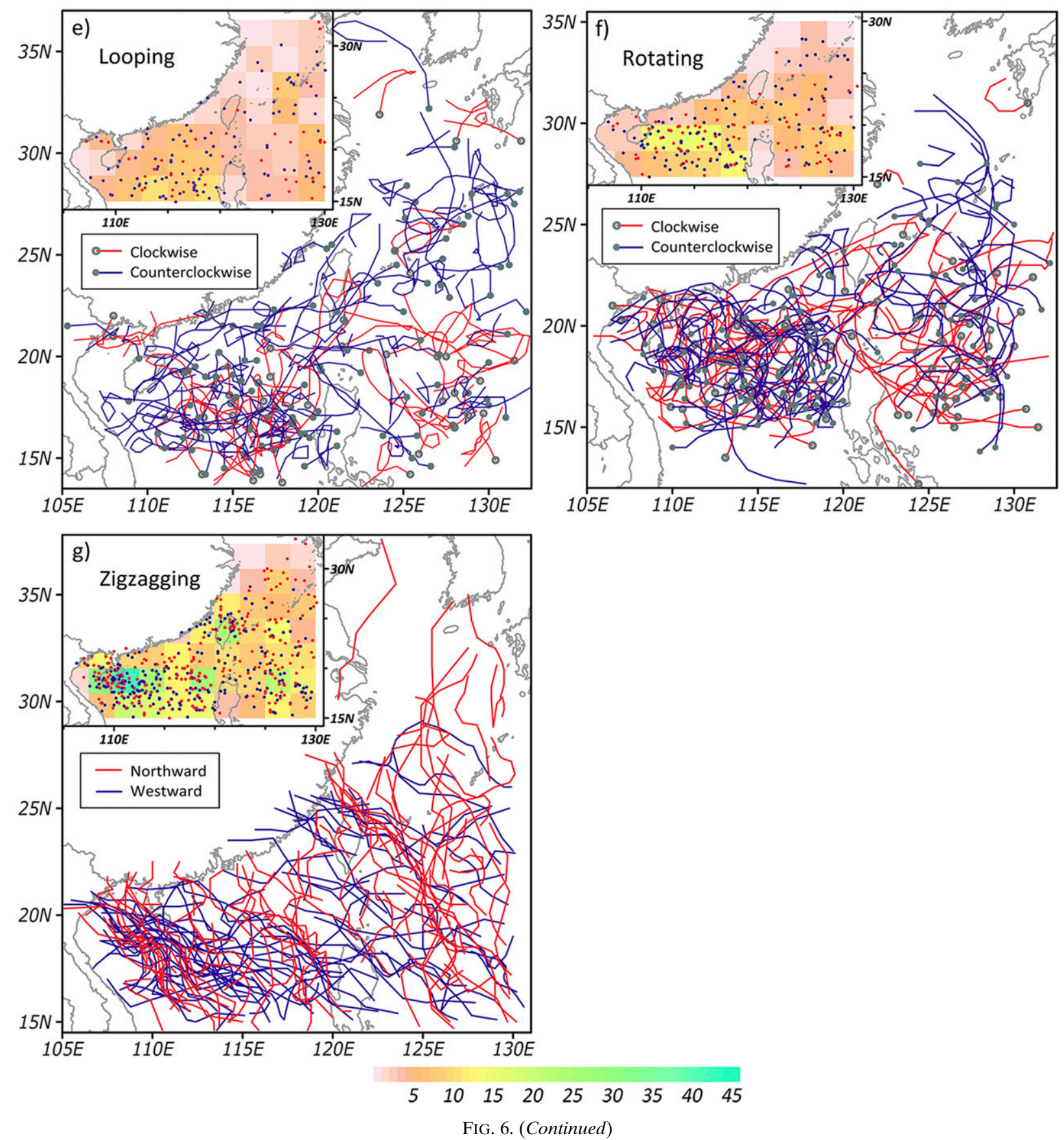

differences between clockwise and counterclockwise tracks. Because of their larger diameters, the mean time scale of the rotating UNTKs is longer than that of the looping ones.

\section{c. Zigzag tracks}

A comparison of Fig. $6 \mathrm{~g}$ with Figs. $6 \mathrm{a}-\mathrm{f}$ indicates that the zigzag-type track has the highest density over most $2.5^{\circ} \times 2.5^{\circ}$ grids, with a peak of 42 inflection points near
Hainan Island. A review of previous studies reveals that some westward- and northward-moving zigzag tracks were closely related to orographic influences (Yeh and Elsberry 1993a,b; Lin et al. 2006; Huang et al. 2011; Peng et al. 2012; Wu et al. 2015) and lower-latitude archshaped monsoon troughs (Lander 1996), respectively. This suggests that the two types of zigzag tracks can be determined by different flow regimes in different regions. In particular, our initial examination of these 
zigzag UNTKs indicates that westward-moving TCs tended to exhibit larger zonal displacements, whereas northward-moving TCs showed larger meridional displacements. So, we classify the zigzag UNTKs in Fig. $6 \mathrm{~g}$ into a westward-moving group (in blue) and a northward-moving group (in red), based on their different displacements.

It is evident that the westward-moving zigzag UNTKs were many fewer in the Luzon Strait but that they tended to cluster around Hainan, and to the west and east of both Taiwan and the Philippines, while the northwardmoving zigzag UNTKs are distributed more uniformly over the study area, except for much-reduced activity on the islands of the Philippines and Taiwan. Note that the zigzag tracks near northern Taiwan were higher in density than those on its southern portions. This result appears to confirm that of Yeh and Elsberry (1993a), who suggested that the upstream track deflections of TCs approaching the southern end of the island are larger and more northward when compared with those of northern-approaching TCs. However, Yeh and Elsberry (1993b) and Lin et al. (2006) found that as a result of the deflection of deep-layer mean flow and weak interaction with the barrier, a northernapproaching TC tends to track continuously around the northern end of the island, while severe distortions of the inner-core circulation and gradual filling of the TC surface center can lead to discontinuous tracks for central-approaching and southern-approaching TCs. Based on their findings, the higher (lower) density of zigzag tracks over the northern (central and southern) part of Taiwan Island could be partly explained by more (less) crossing-island TCs with continuous tracks. However, the opposite is true for Hainan, where highdensity zigzag tracks appeared on its southern side. This could be attributed partly to the topographic influences over the mainland and partly to the binary interactions between TCs or subtropical disturbances along the axis of arch-shaped monsoon troughs, as discussed by Lander (1996). Since the summer monsoon trough generally emerges from East Asia at about $20^{\circ}-25^{\circ} \mathrm{N}$ and extends southeastward to Guam $\left(13^{\circ} \mathrm{N}, 145^{\circ} \mathrm{E}\right)$, Hainan is located near the trough axis where the binary interactions are more frequent.

\section{Summary and conclusions}

In this study, a 65 -yr climatology of UNTKs occurring in the vicinity of China's coastal waters during 1949-2013 is examined using the best-track data archived at the CMA STI in order to bring more attention to the muchneeded research on the processes leading to the development of UNTKs that may influence the coastal regions of China and the other East Asian countries. A total of 1059 UNTK events associated with 564 UTTCs are identified from the usual tracks of 1320 TCs. They are categorized into seven types-sharp westward-turning, sharp eastward-turning, sharp northward-turning, sharp southward-turning, looping, rotating, and zigzagging tracks-based on an improved UNTK classification scheme developed herein. Both the spatial and temporal characteristics of the UNTKs are examined, which represent an extension and update of the previous climatological studies of UNTKs that were performed several decades ago, with the improved UNTK classification scheme and much more up-to-date best-track data.

An analysis of the temporal characteristics shows significant annual variability in the numbers of both total TCs and UTTCs and in the events of total UNTKs. Although most UTTCs are involved with only one type of UNTK or even one UNTK event, TCs with more than one type of UNTK still compose $19 \%$ of total TCs and $45 \%$ of UTTCs, and TCs with more than one UNTK event compose $20 \%$ of total TCs and $46 \%$ of UTTCs. This indicates that some TCs may experience more than one UNTK event in the same or different UNTK types during their life spans. Moreover, most UTTCs appear in June-November, peaking in August. An analysis of their spatial distributions reveals that all seven types of UNTKs tend to take place in the areas to the south of $30^{\circ} \mathrm{N}$, most frequently in the SCS, which may be closely related to the interactions of UTTCs with monsoon circulations [e.g., see Carr and Elsberry (1995) and Wu et al. (2013) for sharp turning tracks; Xu and Gray (1982) for looping and rotating tracks; Lander (1996) for zigzagging tracks]. In addition, since subtropical highs, westerly troughs, upper-tropospheric cold lows, tropical anticyclones, vortex-vortex interaction, internal mesovortices, or intense convective systems and the orographic influences of islands may all play different roles in determining the development of UNTKs, many questions associated with the complicated interactive processes could not be addressed by the present statistical study. Thus, in a forthcoming study, we will perform an in-depth composite analysis of their associated larger-scale circulations in order to gain insight into the driving mechanisms whereby the seven types of UNTKs are generated.

Acknowledgments. We thank Editor Wen-Chau Lee and three anonymous reviewers for their constructive comments, which helped to improve the quality of this paper. This work was supported by the China Scholarship Council, the National Basic Research (973) Program of China (Grants 2014CB441402 and 2015CB452804), the National Natural Science 
Foundation of China (Grants 41275093 and 41475055), the U.S. ONR Grants N000141410143 and N000141712210, and NOAA Grant NA15NWS4680017.

\section{REFERENCES}

Barcikowska, M., F. Feser, and H. von Storch, 2012: Usability of best track data in climate statistics in the western North Pacific. Mon. Wea. Rev., 140, 2818-2830, https://doi.org/10.1175/ MWR-D-11-00175.1.

Burroughs, L. D., and S. Brand, 1973: Speed of tropical storms and typhoons after recurvature in the western North Pacific Ocean. J. Appl. Meteor., 12, 452-458, https://doi.org/10.1175/ 1520-0450(1973)012<0452:SOTSAT $>2.0 . C O ; 2$.

Camargo, S. J., A. W. Robertson, S. J. Gaffney, P. Smyth, and M. Ghil, 2007: Cluster analysis of typhoon tracks. Part I: General properties. J. Climate, 20, 3635-3653, https://doi.org/ 10.1175/JCLI4188.1.

Carr, L. E., III, and R. L. Elsberry, 1995: Monsoonal interactions leading to sudden tropical cyclone track changes. Mon. Wea. Rev., 123, 265-290, https://doi.org/10.1175/ 1520-0493(1995)123<0265:MILTST>2.0.CO;2.

Chan, J. C. L., 1995: Performance of global and regional NWP models in their prediction of Typhoon Nat (1991). Wea. Forecasting, 10, 400-410, https://doi.org/10.1175/ 1520-0434(1995)010<0400:POGARN $>2.0$. CO;2.

- , and H. Lam, 1989: Performance of the ECMWF model in predicting the movement of Typhoon Wayne (1986). Wea. Forecasting, 4, 234-245, https://doi.org/10.1175/ 1520-0434(1989)004<0234:POTEMI >2.0.CO;2.

— W. M. Gray, and S. Q. Kidder, 1980: Forecasting tropical cyclone turning motion from surrounding wind and temperature fields. Mon. Wea. Rev., 108, 778-792, https://doi.org/ 10.1175/1520-0493(1980)108<0778:FTCTMF >2.0.CO;2.

Chen, L. S., and Y. H. Ding, 1979: An Introduction to Typhoons over the Western North Pacific (in Chinese). Science Press, $491 \mathrm{pp}$.

Chu, C. C., 1924: A new classification of typhoons of the Far East. Mon. Wea. Rev., 52, 570-579, https://doi.org/10.1175/ 1520-0493(1924)52<570:ANCOTO>2.0.CO;2.

Elliott, G., and Coauthors, 2014: Motion-Recent advances. Summary Rep. on Eighth Int. Workshop on Tropical Cyclones (IWTC-VIII), 44 pp., http://www.wmo.int/pages/prog/arep/wwrp/ new/documents/Topic1_AdvancesinForecastingMotion.pdf.

Hodanish, S., and W. M. Gray, 1993: An observational analysis of tropical cyclone recurvature. Mon. Wea. Rev., 121, 26652689, https://doi.org/10.1175/1520-0493(1993)121<2665: AOAOTC $>2.0 . \mathrm{CO} ; 2$.

Holland, G. J., 1983: Tropical cyclone motion: Environmental interaction plus a beta effect. J. Atmos. Sci., 40, 328-342, https:// doi.org/10.1175/1520-0469(1983)040<0328:TCMEIP>2.0.CO;2.

_ _ and M. Lander, 1993: The meandering nature of tropical cyclone tracks. J. Atmos. Sci., 50, 1254-1266, https://doi.org/ 10.1175/1520-0469(1993)050<1254:TMNOTC >2.0.CO;2.

Huang, J., J. Du, and W. Qian, 2015: A comparison between a generalized beta-advection model and a classical betaadvection model in predicting and understanding unusual typhoon tracks in eastern China seas. Wea. Forecasting, 30, 771-792, https://doi.org/10.1175/WAF-D-14-00073.1.

Huang, Y.-H., C.-C. Wu, and Y. Wang, 2011: The influence of island topography on typhoon track deflection. Mon. Wea. Rev. 139, 1708-1727, https://doi.org/10.1175/2011MWR3560.1.
Jones, R. W., 1977: Vortex motion in a tropical cyclone model. J. Atmos. Sci., 34, 1518-1527, https://doi.org/10.1175/ 1520-0469(1977)034<1518:VMIATC >2.0.CO;2.

Kitade, T. A., 1981: Numerical study of the vortex motion with barotropic models. J. Meteor. Soc. Japan, 59, 801-807, https:// doi.org/10.2151/jmsj1965.59.6_801.

Kossin, J. P., T. L. Olander, and K. R. Knapp, 2013: Trend analysis with a new global record of tropical cyclone intensity. J. Climate, 26, 9960-9976, https://doi.org/10.1175/ JCLI-D-13-00262.1.

Kuo, H. L., 1950: The motion of atmospheric vortices and the general circulation. J. Meteor., 7, 247-258, https://doi.org/ 10.1175/1520-0469(1950)007<0247:TMOAVA>2.0.CO;2.

_ 1969: Motions of vortices and circulating cylinder in shear flow with friction. J. Atmos. Sci., 26, 390-398, https://doi.org/ 10.1175/1520-0469(1969)026<0390:MOVACC >2.0.CO;2.

Lander, M. A., 1996: Specific tropical cyclone track types and unusual tropical cyclone motions associated with a reverse-oriented monsoon trough in the western North Pacific. Wea. Forecasting, 11, 170-186, https://doi.org/10.1175/ 1520-0434(1996)011<0170:STCTTA > 2.0.CO;2.

Li, X., J. Ming, Y. Wang, K. Zhao, and M. Xue, 2013: Assimilation of T-TREC-retrieved wind data with WRF 3DVAR for the short-term forecasting of Typhoon Meranti (2010) near landfall. J. Geophys. Res. Atmos., 118, $10361-10375$, https://doi.org/10.1002/jgrd.50815.

Liang, J., and L. Wu, 2015: Sudden track changes of tropical cyclones in monsoon gyres: Full-physics, idealized numerical experiments. J. Atmos. Sci., 72, 1307-1322, https://doi.org/ 10.1175/JAS-D-13-0393.1.

,,-- X. Ge, and C.-C. Wu, 2011: Monsoonal influence on Typhoon Morakot (2009). Part II: Numerical study. J. Atmos. Sci., 68, 2222-2235, https://doi.org/10.1175/2011JAS3731.1.

Lin, Y.-L., N. C. Witcraft, and Y.-H. Kuo, 2006: Dynamics of track deflection associated with the passage of tropical cyclones over a mesoscale mountain. Mon. Wea. Rev., 134, 3509-3538, https://doi.org/10.1175/MWR3263.1.

Liu, K. S., and J. C. L. Chan, 2008: Interdecadal variability of western North Pacific tropical cyclone tracks. J. Climate, 21, 4464-4476, https://doi.org/10.1175/2008JCLI2207.1.

Liu, Y., D.-L. Zhang, and M. K. Yau, 1999: A multiscale numerical study of Hurricane Andrew (1992). Part II: Kinematics and inner-core structures. Mon. Wea. Rev., 127, 2597-2616, https://doi.org/10.1175/ 1520-0493(1999)127<2597:AMNSOH>2.0.CO;2.

Nof, D., 1985: Joint vortices, eastward propagating eddies and migratory Taylor columns. J. Phys. Oceanogr., 15, 1114-1137, https://doi.org/ 10.1175/1520-0485(1985)015<1114:JVEPEA > 2.0.CO;2.

Peng, L., S.-T. Wang, S.-L. Shieh, M.-D. Cheng, and T.-C. Yeh, 2012: Surface track discontinuity of tropical cyclones crossing Taiwan: A statistical study. Mon. Wea. Rev., 140, 121-139, https://doi.org/ 10.1175/MWR-D-10-05050.1.

Peng, M. S., D.-S. Chen, S. W. Chang, C.-P. Chang, and B.-F. Jeng, 1995: Improvement of numerical prediction of typhoon tracks in the western North Pacific basin near Taiwan. Wea. Forecasting, 10, 411-424, https://doi.org/10.1175/ 1520-0434(1995)010<0411:IONPOT>2.0.CO;2.

Rappaport, E. N., and Coauthors, 2009: Advances and challenges at the National Hurricane Center. Wea. Forecasting, 24, 395-419, https://doi.org/10.1175/2008WAF2222128.1.

Riehl, H., 1954: Tropical Meteorology. McGraw-Hill, 392 pp.

- and R. J. Shafer, 1944: The recurvature of tropical storms. J. Meteor., 1, 42-54, https://doi.org/10.1175/1520-0469(1944)001<0001: TROTS $>2.0 . \mathrm{CO} ; 2$ 
Shi, W., J. Fei, X. Huang, X. Cheng, and J. Ding, 2014: A numerical study on the combined effect of midlatitude and low-latitude systems on the abrupt track deflection of Typhoon Megi (2010). Mon. Wea. Rev., 142, 2483-2501, https://doi.org/10.1175/MWR-D-13-00283.1.

Torn, R. D., and C. Snyder, 2012: Uncertainty of tropical cyclone besttrack information. Wea. Forecasting, 27, 715-729, https://doi.org/ 10.1175/WAF-D-11-00085.1.

Visher, S. S., 1922: Notes on typhoons, with charts of normal and aberrant tracks. Mon. Wea. Rev., 50, 583-589, https://doi.org/ 10.1175/1520-0493(1922)50<583:NOTWCO > 2.0.CO;2.

Wei, N., Y. Li, D.-L. Zhang, Z. Mai, and S.-Q. Yang, 2016: A statistical analysis of the relationship between uppertropospheric cold low and tropical cyclone track and intensity change over the western North Pacific. Mon. Wea. Rev., 144, 1805-1822, https://doi.org/10.1175/MWR-D-15-0370.1.

Willoughby, H. E., 1988: Linear motion of a shallow-water, barotropic vortex. J. Atmos. Sci., 45, 1906-1928, https://doi.org/ 10.1175/1520-0469(1988)045<1906:LMOASW>2.0.CO;2.

Wu, C.-C., T.-S. Huang, W.-P. Huang, and K.-H. Chou, 2003: A new look at the binary interaction: Potential vorticity diagnosis of the unusual southward movement of Tropical Storm Bopha (2000) and its interaction with Supertyphoon Saomai (2000). Mon. Wea. Rev., 131, 1289-1300, https://doi.org/10.1175/1520-0493(2003)131<1289: ANLATB $>2.0 . \mathrm{CO} ; 2$.

, S.-G. Chen, C.-C. Yang, P.-H. Lin, and S. D. Aberson, 2012: Potential vorticity diagnosis of the factors affecting the track of Typhoon Sinlaku (2008) and the impact from dropwindsonde data during T-PARC. Mon. Wea. Rev., 140, 2670-2688, https://doi.org/10.1175/MWR-D-11-00229.1.

-, T.-H. Li, and Y.-H. Huang, 2015: Influence of mesoscale topography on tropical cyclone tracks: Further examination of the channeling effect. J. Atmos. Sci., 72, 3032-3050, https:// doi.org/10.1175/JAS-D-14-0168.1.

Wu, C.-R., Y.-L. Chang, L.-Y. Oey, C.-W. J. Chang, and Y.-C. Hsin, 2008: Air-sea interaction between Tropical Cyclone Nari and Kuroshio. Geophys. Res. Lett., 35, L12605, https://doi.org/ 10.1029/2008GL033942.

Wu, L., J. Liang, and C.-C. Wu, 2011a: Monsoonal influence on Typhoon Morakot (2009). Part I: Observational analysis. J. Atmos. Sci., 68, 2208-2221, https://doi.org/10.1175/2011JAS3730.1.

- H. Zong, and J. Liang, 2011b: Observational analysis of sudden tropical cyclone track changes in the vicinity of the East China Sea. J. Atmos. Sci., 68, 3012-3031, https://doi.org/ 10.1175/2010JAS3559.1.
_ _ Z. Ni, J. Duan, and H. Zong, 2013: Sudden tropical cyclone track changes over the western North Pacific: A composite study. Mon. Wea. Rev., 141, 2597-2610, https://doi.org/ 10.1175/MWR-D-12-00224.1.

Xu, J., and W. M. Gray, 1982: Environmental circulations associated with tropical cyclones experiencing fast, slow and looping motion. Colorado State University Atmospheric Science Paper 346, 273 pp.

Yang, C.-C., C.-C. Wu, K.-H. Chou, and C.-Y. Lee, 2008: Binary interaction between Typhoons Fengshen (2002) and Fungwong (2002) based on the potential vorticity diagnosis. Mon. Wea. Rev., 136, 4593-4611, https://doi.org/ 10.1175/2008MWR2496.1.

Yang, M.-J., D.-L. Zhang, and H.-L. Huang, 2008: A modeling study of Typhoon Nari (2001) at landfall. Part I: Topographic effects. J. Atmos. Sci., 65, 3095-3115, https://doi.org/10.1175/ 2008JAS2453.1.

Yeh, T. C., 1950: The motion of tropical storms under the influence of a superimposed southerly current. J. Meteor., 7, 108-113, https://doi.org/10.1175/1520-0469(1950)007<0108: TMOTSU $>2.0 . \mathrm{CO} ; 2$.

Yeh, T.-C., and R. L. Elsberry, 1993a: Interaction of typhoons with the Taiwan orography. Part I: Upstream track deflections. Mon. Wea. Rev., 121, 3193-3212, https://doi.org/10.1175/ 1520-0493(1993)121<3193:IOTWTT>2.0.CO;2.

$\longrightarrow$, and 1993b: Interaction of typhoons with the Taiwan orography. Part II: Continuous and discontinuous tracks across the island. Mon. Wea. Rev., 121, 3213-3233, https://doi.org/ 10.1175/1520-0493(1993)121<3213:IOTWTT>2.0.CO;2.

Ying, M., W. Zhang, H. Yu, X. Lu, J. Feng, Y. Fan, Y. Zhu, and D. Chen, 2014: An overview of the China Meteorological Administration tropical cyclone database. J. Atmos. Oceanic Technol., 31, 287-301, https://doi.org/10.1175/ JTECH-D-12-00119.1.

Zhang, D.-L., L. Tian, and M.-J. Yang, 2011: Genesis of Typhoon Nari (2001) from a mesoscale convective system. J. Geophys. Res., 116, D23104, https://doi.org/10.1029/ 2011JD016640.

Zhao, K., X. Li, M. Xue, B. J.-D. Jou, and W.-C. Lee, 2012: Short-term forecasting through intermittent assimilation of data from Taiwan and mainland China coastal radars for Typhoon Meranti (2010) at landfall. J. Geophys. Res. Atmos., 117, D06108, https://doi.org/ 10.1029/2011JD017109. 